\section{§3. Extraction of Hydrogen into Vacuum by Electrochemical Hydrogen Pump for Hydrogen Isotope Recovery}

Tanaka, M.

In a fusion reaction, the exhaust gas processing system must achieve a high tritium decontamination factor. From the viewpoints of fuel balance and safety, tritium loss from the exhaust gas processing system should be minimized. Accordingly, the development of fuel cycle technology is a critical issue for realizing a fusion reactor.

To recovery hydrogen isotope gas from vacuum exhaust, the application of an electrochemical hydrogen pump using a proton conductor that transports protons in ceramics at high temperatures have been proposed. This type of electrochemical hydrogen pump can extract and recover molecular tritium from gas mixtures containing tritiated hydrocarbons and water vapor. To recovery pure hydrogen isotope gas for the fuel cycle, however, the hydrogen pump must be operated under vacuum to avoid contamination from the purge gas. Yet the characteristics of the hydrogen pump under vacuum are not fully known.

In this report, hydrogen transport characteristics under vacuum are analyzed by electrochemical impedance spectroscopy (EIS) using an equivalent circuit model and then the results for two types of electrodes - pasted and electroless plated electrodes - are compared. EIS is a technique for characterizing electrochemical systems. A simple and widely used semi-empirical model of an electrochemical cell is the Randles circuit which describes the response of a single-step charge-transfer process with diffusion. The simplified equivalent circuit consists of an electrolyte bulk resistor $\left(\mathrm{R}_{\mathrm{b}}\right)$ in series with the parallel combination of the double-layer capacitance $\left(\mathrm{C}_{\mathrm{g} . \mathrm{b}}\right)$ and the grain boundary resistor $\left(\mathrm{R}_{\mathrm{g} . \mathrm{b}}\right)$. and in series with the parallel combination of the charge transfer capacitance $\left(\mathrm{C}_{\mathrm{ct}}\right)$ and the Faraday impedance $\left(\mathrm{Z}_{\mathrm{F}}\right)$, which is due to processes such as chemical reactions, mass transfer, and adsorption around the electrode. Taking into consideration the elementary processes in a proton conductor and on its surface, the Faraday impedance consists of the charge transfer resistor $\left(\mathrm{R}_{\mathrm{ct}}\right)$ and the finite-length Warburg impedance with a short circuit terminus $\left(\mathrm{Z}_{\mathrm{Ws}}\right)$, in series, as shown in Fig. 1 . Accordingly, the combined impedance $(Z)$ of the equivalent circuit is expressed as follow:

$$
\mathrm{Z}=\mathrm{R}_{\mathrm{b}}+\frac{1}{\frac{1}{\mathrm{R}_{\text {g.b. }}}+\mathrm{j} \omega \mathrm{C}_{\text {g.b }}}+\frac{1}{\frac{1}{\mathrm{R}_{\mathrm{ct}}+\mathrm{Z}_{\mathrm{w}_{\mathrm{s}}}}+j \omega \mathrm{C}_{\mathrm{ct}}},
$$

where $\mathrm{j}$ is the imaginary unit and $\omega$ is angular frequency. To determine the impedance parameters in Fig. 1, the impedance spectra data were analyzed under the equivalent circuit model by using EIS Spectrum Analyzer software. In Fig. $1, Z_{W s}$ is the solution of the one-dimensional diffusion equation of a particle, which is analogous to wave transmission in a finite-length $\mathrm{RC}$ transmission line:

$$
\mathrm{Z}_{\mathrm{w}_{\mathrm{s}}}=\frac{\mathrm{W}_{\mathrm{Sr}}}{\sqrt{\omega}}(1-\mathrm{j}) \tanh \left[\mathrm{W}_{\mathrm{Sc}} \sqrt{\mathrm{j} \omega}\right]
$$

where $\mathrm{W}_{\mathrm{Sr}}$ is equal to the Warburg coefficient, which is proportional to $1 / \mathrm{D}^{0.5} ; \mathrm{W}_{\mathrm{Sc}}$ is expressed as $\mathrm{d} / \mathrm{D}^{0.5} ; \mathrm{d}$ is Nernst diffusion layer thickness; and $\mathrm{D}$ is effective diffusivity of gas through the porous layer.

The impedance parameters obtained by EIS analysis are summarized in Fig. 2. The bulk resistance and grain boundary resistance were independent of pressure in the investigated pressure range. In the case of the plated electrode, the impedance parameter $\mathrm{W}_{\mathrm{Sr}}$ was higher than the other impedance parameters and reached a minimum around $10 \mathrm{kPa}$. Since the impedance parameters $\mathrm{W}_{\mathrm{Sc}}$ and $\mathrm{W}_{\mathrm{Sr}}$ are proportional to $\mathrm{D}^{-0.5}$, the gas transport in the plated electrode should be suppressed even under vacuum because of the plated electrode microstructure. In contrast, in the case of the pasted electrode, the impedance parameter $\mathrm{W}_{\mathrm{Sc}}$ at atmospheric pressure was much higher than that under vacuum, and it was much higher than the other impedance parameters. Furthermore, $\mathrm{W}_{\mathrm{Sc}}$ slightly increased under vacuum pressure less than $0.1 \mathrm{kPa}$. The gas would be relatively diffuse in the pasted electrode under vacuum, because the pasted electrode has large pores.

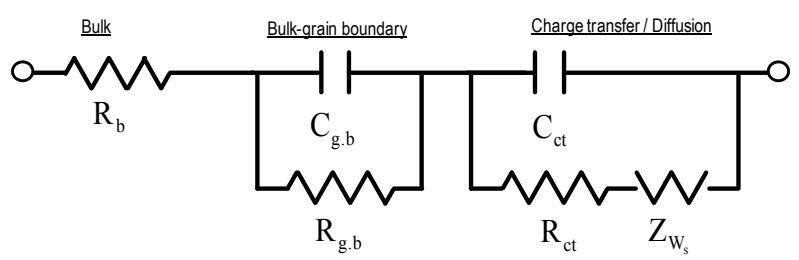

Fig. 1. Equivalent circuit based on Randles circuit model.
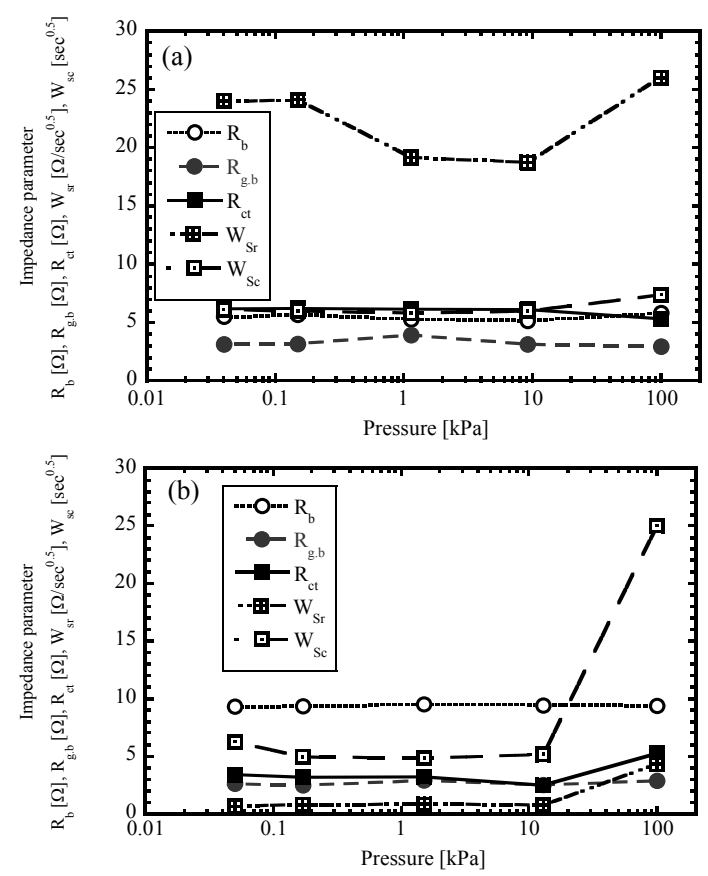

Fig. 2. Dependence of impedance parameters on total pressure: (a) plated electrode and (b) pasted electrode. 